\title{
The effect of acid treatment on the surface chemistry and topography of graphite.
}

Emir Bouleghlimat, Philip R. Davies ${ }^{*}$, Robert J. Davies,

Rebecca Howarth, Jiri Kulhavy, David J. Morgan

Cardiff Catalysis Institute, School of Chemistry, Cardiff University

\begin{abstract}
Highly oriented pyrolytic graphite (HOPG) samples were investigated as model catalyst supports. The surfaces were treated with dilute $\mathrm{HCl}$ and $\mathrm{HNO}_{3}$ under ambient conditions and examined with atomic force microscopy and scanning tunnelling microscopy (STM) and Xray photoelectron spectroscopy (XPS). Raised features were formed on the HOPG surface after acid treatment. These protrusions were typically 4-6 $\mathrm{nm}$ in height and between 10 to 100 $\mathrm{nm}$ in width, covering $5 \%$ to $20 \%$ of the substrate for acid concentrations between 0.01 and $0.2 \mathrm{M}$. Both width and surface density of the features increases with acid concentration but the heights are not affected. STM images show that the graphite lattice extends over the protrusions indicating that the features are "blisters" on the surface rather than deposited material, a view that is supported by the XPS which shows no other significant adsorbates except for oxygen in the case of the nitric acid. We propose that penetration of the acid at defective sites leads to a decrease in the interplanar van der Waals forces and a local delamination similar to the "bubbles" reported between exfoliated graphene sheets and a substrate. These findings are important in the context of understanding how carbon supports stabilise active components in heterogeneous catalysts.
\end{abstract}

\section{Introduction}

Carbon materials, including graphite and "activated carbon", are commonly used as a supports for heterogeneous catalysts because of their relatively low cost, high surface area and the ease with which precious metals can be reclaimed from the support at the end of the catalyst's active life.[1-3] However, in nearly all cases, carbon supports are acid washed, typically with $\mathrm{HCl}$ and/or $\mathrm{HNO}_{3}$, before the adsorption of the active component. The wash has two purposes; it removes unwanted contaminants such as $\mathrm{N}, \mathrm{P}, \mathrm{Si}, \mathrm{Ca}, \mathrm{Na}, \mathrm{K}, \mathrm{Al}, \mathrm{Zn}$ and Fe, that accumulate on the carbon surface during preparation; and it is also thought to introduce hydrophilic functional groups that modify the behaviour of the carbon towards the adsorption of the active component and the reaction solvent, [4,5]. There have been a number of studies of the effects of acid washing on carbons; Wang et al. for example, showed [5] that the use of an acid wash significantly increased the dispersion of nickel on carbon which they

\footnotetext{
* Corresponding author. Tel/Fax: +44 (0)292087 4072. E-mail address: daviespr@cf.ac.uk (P.R. Davies)
} 
attributed to the formation of acidic functional groups that could react with the nickel salts. They noted that treatment with $\mathrm{HCl}$ resulted in a less stable catalyst than with nitric acid and also reported an increase in pore volume and surface area. A later paper by Wang[6] gives further detail of the differences between $\mathrm{HCl}$ and $\mathrm{HNO}_{3}$ treatments, this time in relation to the adsorption of dye molecules. Using temperature programmed desorption (TPD), the authors observed differences in the evolution of $\mathrm{CO}_{2}$ and $\mathrm{CO}$ from the differently treated carbons with a $\mathrm{HNO}_{3}$ wash giving rise to significantly more $\mathrm{CO}_{2}$ desorption at lower temperatures. The authors accounted for this behaviour by suggesting that the nitric acid gives rise to carboxylic, anhydride, lactone and phenol groups. In contrast, $\mathrm{HCl}$ washed carbon gave only a small change in $\mathrm{CO}_{2}$ desorption from that seen with the untreated surface. Wang and Zhu suggest that this indicates that the $\mathrm{HCl}$ causes a small decrease in acidic groups at the surface. However, a sharp increase in $\mathrm{CO}$ desorption at high temperatures is also observed after $\mathrm{HCl}$ treatment which was attributed by the authors to an increase in phenol groups. There is some support for these observations in their x-ray photoelectron (XP) spectra of the $\mathrm{C}(1 \mathrm{~s})$ region but unfortunately no $\mathrm{O}(1 \mathrm{~s})$ data are provided to confirm the assignments. Zhu, Radovic and Lu have published a more detailed discussion of the effects of acid washes on carbon supports, where they examined the effect of $\mathrm{HCl}, \mathrm{HNO}_{3}$ and $\mathrm{HF}$ treatments on $\mathrm{NO}$ and $\mathrm{NO}_{2}$ reduction by carbon-supported copper catalysts.[7] In their paper they draw attention to two separate phenomena which may play a role in catalysis over acid washed carbon catalysts. Firstly, the increase in $-\mathrm{CO}_{2}{ }^{-}$functionality can hinder the adsorption of anionic species because of charge repulsion whilst improving the adsorption of cations; thus $\mathrm{PtCl}_{6}{ }^{2-}$ adsorption is adversely affected by $\mathrm{HNO}_{3}$ treatment whilst $\mathrm{Cu}^{2+}$ adsorption is enhanced. Secondly, the presence of - $\mathrm{CO}$ groups on the surface act as stabilising sites which hinder sintering during calcination. Acid pre-treatment of carbon nanotubes used as supports for iron based Fischer-Tropsch catalysts resulted in smaller iron particles anchored on the support [8] as well as increasing the overall surface area and introducing defects into the nanotubes.

More recently Conte et al. have studied [9,10] the effects of acid washing carbon supported gold catalysts in relation to the gold/carbon catalyst they have pioneered for the hydrochlorination of acetylene, an emerging alternative to the oxychlorination of ethene currently used industrially for the synthesis of vinyl chloride monomer (VCM). Since 1985, Hutchings' group [11-14] have investigated the possibility of replacing the current environmentally unfriendly $\mathrm{Hg}$ based catalyst with a $\mathrm{Au} / \mathrm{C}$ alternative. A key component of this development has been the use of acid treatments before impregnation with gold and also after reaction to regenerate the catalyst [15]. Conte et al. show how the acid treatment has a direct effect on both the chemistry and the structure of the carbon supports. They conclude 
that the $\mathrm{Au} / \mathrm{C}$ interface may be as important to hydrochlorination catalysis as the $\mathrm{Au} / \mathrm{Oxide}$ interface is to the oxidation of $\mathrm{CO}$.

Our particular interest in this topic is how the treatment of the carbon surfaces and the functional groups that are formed as a result, affects the nature of the gold nanoparticles that are deposited to form the working catalyst. To study these phenomena at the nanoscale we have employed a combination of atomic force microscopy (AFM) and scanning tunnelling microscopy (STM), together with XP spectroscopy. The microscopy methods require relative flat surfaces and therefore our investigations are carried out on highly oriented pyrolytic graphite (HOPG). HOPG is often used as a model carbon surface for studies under ultra high vacuum conditions[16-18] despite the lack of functional groups that would exist on real catalyst surfaces. For this reason the present study can also be regarded as bridge between the idealised UHV systems and the practical catalysts.

\section{Experimental}

The HOPG samples ( $10 \mathrm{~mm}$ square, ZYH quality, NT-MDT) were cleaned before each experiment by peeling off the top few graphene monolayers using adhesive tape. Acid treatment involved placing a droplet $(100 \mu \mathrm{l})$ of acid $\left(\mathrm{HCl}\right.$ or $\left.\mathrm{HNO}_{3}\right)$ and leaving to stand for a set period of time before drying under a pure He stream. The acids wet the surface quite effectively and therefore no investigation was made into the effect of droplet size. Acid concentrations of $0.01,0.1$ and $0.2 \mathrm{M}$ were investigated, and ultra pure water (Millipore) was used for dilution and as a control experiment, Figure 1 (c), after initial experiments showed dionized water left surfaces contaminated with calcium and sodium containing deposits.

AFM images were acquired on a Bruker Veeco Multimode probe in tapping mode using silicon nitride tips. STM was performed under ambient conditions on a bench top Nanosurf Easyscan apparatus using a platinum-iridium tip. All SPM images were analysed using WSxM software; the tools available within this software are discussed elsewhere.[19] XP spectra were recorded with a Kratos Axis Ultra-DLD photoelectron spectrometer using a monochromatic $\mathrm{Al} \mathrm{K} \alpha \mathrm{x}$-ray source and the "hybrid spectroscopy" mode resulting in an analysis area of $700 \times 400 \mu \mathrm{m}$ at a pass energy of $40 \mathrm{eV}$ for high resolution scans and $160 \mathrm{eV}$ for survey scans. All binding energies are referenced to the $\mathrm{C}(1 \mathrm{~s})$ peak at $284.3 \mathrm{eV}$. Surface concentration have been calculated using the method of described in detail by Carley et al. $[20,21]$

\section{Results}

\subsection{AFM of clean and water treated HOPG}

AFM images of clean (freshly stripped) HOPG surface show large flat terraces up to 1.5 $\mu \mathrm{m}$ in width separated by steps which are up to $15 \mathrm{~nm}$ in height, (fig. 1a \& b). Washing with 
dionised water resulted in deposits of sodium and calcium containing materials but control experiments involving HOPG treated with ultra-pure water retain the large flat terraces with no evidence for the development of other features, (fig. $1 \mathrm{c}$ ).

\subsection{AFM of acid treated HOPG}

Even relatively weak acids have an immediate impact on the HOPG surface. Figure 2 shows AFM images of $\mathrm{HOPG}$ treated with $0.01 \mathrm{M} \mathrm{HNO}_{3}$ and $\mathrm{HCl}$ for 1 minute and for 30 minutes. After the one minute treatment, protrusions are evident in the AFM images of both surfaces with heights of $\sim 1.5 \mathrm{~nm}$ and widths of approximately $25 \mathrm{~nm}$. In the case of the $\mathrm{HCl}$ there appears to be a preponderance of raised features at the step edges although they are also seen in the middle of terraces. For the nitric acid treated surface the distribution is more random. After the longer exposure to the acid the number of features on both surfaces has increased and as the line profiles show, there is also a small increase in both height and width of the features. There remains a slight tendency for the protrusions to be located at step edges but this is by no means the only site, may features are located in the centre of terraces. Note also that the AFM images exaggerate the z-axis significantly, an effect exacerbated by the different $\mathrm{x}$ and $\mathrm{y}$ scales used in the profile plots in order to emphasise the features present in the data. The diameters of the protrusions are typically ten times larger than the heights indicating gentle mounds rather than the hemispherical particles the images suggest.

More severe treatments give rise to greater numbers of features with larger dimensions. Figure 3 (a \& b), shows the consequence of treating a surface with $0.1 \mathrm{M} \mathrm{HCl}$ for 30 minutes. The surface is decorated with raised features of between 20 and $40 \mathrm{~nm}$ in diameter and up to $7 \mathrm{~nm}$ in height. The features are non-uniform in circumference with no preference to form circular shapes or mimic the structure of the HOPG substrate. The surface area covered by the features was estimated from flooding analysis to be between 10 and $15 \%$. There is an increase in size and number of features at the higher $\mathrm{HCl}$ concentration of $0.2 \mathrm{M}$ (Fig $3 \mathrm{c} \&$ d) with feature widths increasing to $\sim 60 \mathrm{~nm}$ and the coverage to approximately $20 \%$ but the height of the protrusions is not significantly affected.

$\mathrm{HCl}$ is a strong acid $\left(\mathrm{pK}_{\mathrm{a}}\right.$ of -7) but $\mathrm{HOPG}$ treated with the less acidic $\mathrm{HNO}_{3}\left(\mathrm{pK}_{\mathrm{a}}\right.$ in the range -1 to -2 ) showed very similar behaviour. AFM images after treatment with $0.1 \mathrm{M}$ $\mathrm{HNO}_{3}$ (fig. 4 a-c) show protrusions at the surface with widths typically between 30 and 45 $\mathrm{nm}$ but with some features as much as $60 \mathrm{~nm}$ across. The heights of these features were measured to be approximately $5 \mathrm{~nm}$. Figure $4 \mathrm{~b}$ shows that in some areas the density of raised features is less than in others but in this case the structures are somewhat larger in diameter. The coverage of the HOPG surface by the protrusions was estimated to be around $8 \%$. As with $\mathrm{HCl}$, increasing the acid concentration to $0.2 \mathrm{M}$, led to an increase in the size of the protrusions which were predominantly in the range 40 to $60 \mathrm{~nm}$ with some larger features as 
much as $75 \mathrm{~nm}$ in diameter. It was estimated that at $0.2 \mathrm{M}$ the features occupied around $15 \%$ of the substrate surface.

We have investigated the stability of the features towards further treatment with water. Washing in ultrapure water for 1 minute followed by drying in helium resulted in no change in the AFM images for either the nitric or hydrochloric acid treated surfaces.

Quantification of the images, using the flooding analysis tool of WSxM,[19] confirms the qualitative discussion above and is illustrated in Figure 5 which compares the distribution of raised features over similar areas of graphite after treatment with acids of different strength. Both $\mathrm{HCl}$ and $\mathrm{HNO}_{3}$ give similar effects with the nitric acid producing slightly more protrusions. In both cases, the height of the protrusions is affected less by the acid concentration than the number of features, particularly smaller features which, increases with acid strength together with the range of "diameters" of the protrusions. This is consistent with a model in which larger islands are formed by the coalescence of smaller protrusions a conclusion that is also hinted at by the observed shape of the islands in the AFM images which are reminiscent of the joining of liquid drops on the surface.

\subsection{XPS of acid treated HOPG}

XP spectra of the acid treated HOPG were recorded (Figure 6) with the aim of detecting any functional groups generated on the surface by the acid treatment. In the $\mathrm{C} 1 \mathrm{~s}$ region, species such as hydroxyl and ester or carbonyl would be expected at between 286-288 eV and 287-290 eV respectively[22] but no significant additional components are observed to the high binding energy side of the main $\mathrm{C} 1 \mathrm{~s}$ peak at $284.3 \mathrm{eV}$ indicating that if these are formed their concentrations are low, probably less than $\sim 4 \times 10^{13} \mathrm{~cm}^{-2}(\sim 1 \%$ of a monolayer $)$.

From the O1s spectra it is evident that a small amount of oxygen is present at the clean surface giving a weak, broad $\mathrm{O} 1 \mathrm{~s}$ signal ranging from $531.5 \mathrm{eV}$ to $\sim 534.5 \mathrm{eV}$ and a total oxygen surface concentration (calculated from the XP peak area ) corresponding to ca. $7.5 \times 10^{13} \mathrm{~cm}^{-2}$. This is approximately $2 \%$ of the carbon atom concentration at the clean surface $\left(3.9 \times 10^{15} \mathrm{~cm}^{-2}\right)$ and is typical of HOPG samples cleaved in air. Treatment with $\mathrm{HCl}$ caused an increase in the surface oxygen concentration to $2.1 \times 10^{14} \mathrm{~cm}^{-2}$ equivalent to ca. $5 \%$ of a monolayer with the binding energy centred at $531.8 \mathrm{eV}$. This increase has been attributed previously[23] to the binding of hydroxide ions to the vacant sites at the defects created by the $\mathrm{HCl}$ in the graphene layer. $\mathrm{HNO}_{3}$ treatment resulted in a greater increase in surface oxygen concentration than did the $\mathrm{HCl}$, with a calculated concentration of $5.3 \times 10^{14} \mathrm{~cm}^{-2}$ equivalent to ca. $14 \%$ of a monolayer. The O1s binding energy was again centred at 531.8 $\mathrm{eV}$. After treatment with $\mathrm{HCl}$ a small $\mathrm{Cl} 2 \mathrm{p}$ signal appears at approximately $197 \mathrm{eV}$ with a peak area corresponding to a surface concentration of $4.2 \times 10^{13} \mathrm{~cm}^{-2}$ equivalent to $\sim 1 \%$ monolayer. The $\mathrm{N}$ 1s spectrum of the clean graphite surface exhibits a small peak at a binding 
energy of $\sim 399.5 \mathrm{eV}$, but on treatment with $\mathrm{HNO}_{3}$ another, weaker peak appears at $406 \mathrm{eV}$, a binding energy which is typical of a functional group such as nitrate. The increase in nitrogen concentration corresponds to $\sim 0.7 \%$ of a monolayer, $\left(\sim 2.7 \times 10^{13} \mathrm{~cm}^{-2}\right)$. No other elements were detected in the survey scans for any of the surfaces. In particular, the presence of sodium and calcium can be ruled out.

\section{$3.4 \quad$ STM of acid treated HOPG}

The atomic force microscope used in this study is limited to a resolution of $\sim 2 \mathrm{~nm}$, therefore to investigate the nature of the features generated on the graphite surface in more detail we examined the HOPG samples with a desktop STM capable of atomic resolution. STM images of freshly cleaved and $\mathrm{HNO}_{3}$ treated HOPG surfaces are shown in figure 7. At the clean surface the characteristic C-C interatomic spacing of $1.4 \AA$ can be identified [24], although individual atoms were not imaged (Figure 7a). A typical STM image of a nitric acid induced feature is shown at large scale in Figure $7 b$ and in close up in Figure $7 c$ where the characteristic C-C lattice spacing is observed on top of the feature. This demonstrates that the graphite lattice extends over the protrusions - although atomic size defects could still be present. Similar images were recorded at different scan rates in order to rule out external interference and repeated on other features in separate experiments.

\section{Discussion}

Our aim in treating the HOPG with acids was to generate functional groups at the surface and to study how these influence the deposition of the gold nanoparticles involved in the active hydochlorination catalyst. However, the XPS data presented here shows little functionlisation of the surface was occurring under the mild conditions used, whereas the AFM data shows significant impact on the surface topography. The nature of the features produced is therefore the central focus of this paper. In view of the XPS data it is unlikely that the protrusions seen in AFM could be due to the deposition of some precipitate from solution: sodium and calcium are frequent contaminants in lower quality water (as shown in the control experiments involving deionised water) but neither was seen in any spectra of these samples and the sensitivity of the XPS is sufficient to rule out their presence here. Furthermore, deposition on HOPG surfaces is typically dominated by nucleation at step edges and the features observed in the AFM are more evenly dispersed over the surface, albeit a slight preponderance at step edges for $\mathrm{HCl}$. An alternative explanation is that the acid treatment results in a degradation of the carbon lattice, possibly at existing defect sites, and that these disrupted sites are imaged as raised features. This interpretation is also contradicted by the XPS data which indicates a lower surface concentration of functional groups at the surface than we would anticipate for the surface coverage of features we observe. It is the 
STM data however which is the strongest evidence against either of the previous two explanations showing as it does a largely coherent graphite lattice extending over the protrusions seen in the STM and AFM images. This data leads us to propose that the raised features are due to local areas of delamination.

Interestingly, there is some similarity between the features observed here and the "bubbles" reported to form between single graphene layers placed on a variety of different substrates.[25,26] Bubble diameters ranging from $10 \mathrm{~nm}$ to 10 's of microns have been reported but their origin is not fully understood. One suggestion is that they are caused by residual gas trapped between the graphene and the substrate on which the layer is deposited. Since graphene is impermeable to other molecules the protrusions seen in the present case imply the presence or formation of defects in the graphene sheet. If such defects are formed by the acid treatment it is difficult to explain why any gases generated between the graphene sheets could not escape through the defects and therefore we attribute the protrusions seen here to local weakening of the interplanar forces caused by disruption of the bonding by reaction with the acids penetrating through the graphene. Disruption of the graphene lattice by $\mathrm{HCl}$ has been reported previously albeit using much more severe conditions than those used in this study (e.g. $35 \% \mathrm{HCl}, 90{ }^{\circ} \mathrm{C}, 4$ hours[23]) and Shin et al. have suggested "directacid-penetration" through graphitic layers with a nitric acid/sulphuric acid mixture.[27]

Graphite intercalation as a result of mild aqueous acid electrolytes has also been reported previously [28-30] but in these cases, is driven by large applied potentials to initiate the reaction. The present results are the first to indicate that even mild treatment of graphite surfaces can have significant effects on the surface topography. Interestingly, Conte et al. observed [10] a significant degree of penetration of gold into the carbon lattice during acid treatment and suggest that the activity of the $\mathrm{Au}^{3+}$ in the hydrochlorination reaction may depend as much on where it is on the catalyst as what it is. In future work we will investigate how the modifications to the surface observed here can influence the topography and nature of the gold nanoparticles deposited on these surfaces.

\section{Conclusions}

The treatment of HOPG surfaces with $\mathrm{HCl}$ and $\mathrm{HNO}_{3}$ has a significant impact on the surface topography, generating blister like features which adorn the surface even after a brief acid treatment at low acid concentration. XPS results give strong evidence against the presence of functional groups at high enough concentrations to be responsible for these features and STM studies at atomic resolution confirm that the graphite lattice extends over the protrusions. We conclude that this modification of the HOPG surface occurs through intrusion by the acid into the graphite, causing weakening and breakage of the interplanar bonds. The results may have implications for our understanding of carbon based catalysts 
since functional groups generated under the surface would be less effective at stabilising nanoparticles of active material deposited onto the graphite.

\section{Acknowledgements}

The authors are grateful to Dr Marco Conte for discussions concerning the gold/carbon hydrochlorination catalyst and for copies of references $[9,10]$ The research was supported by EPSRC grant EP/I038748/1. EB thanks Cardiff University for provision of a summer studentship under the CUROP scheme. The authors would also like to thank the two editors in chief (Profs Thrower \& Hurt) for their help in getting this paper to publication.

\section{References}

1. Hutchings G, Carrettin S, Landon P, Edwards J, Enache D, Knight D, et al. New approaches to designing selective oxidation catalysts: $\mathrm{Au} / \mathrm{C}$ a versatile catalyst. Top. Catal. 2006;38(4):223-30.

2. Rodríguez-reinoso F. The role of carbon materials in heterogeneous catalysis. Carbon. 1998;36(3):159-75.

3. Figueiredo J., Pereira MF., Freitas MM., Órfão JJ. Modification of the surface chemistry of activated carbons. Carbon. 1999;37(9):1379-89.

4. Albers P, Deller K, Despeyroux BM, Prescher G, Schafer A, Seibold K. SIMS/XPS Investigations on Activated Carbon Catalyst Supports. J. Catal. 1994;150(2):368-75.

5. Wang S, Lu GQ. Effects of acidic treatments on the pore and surface properties of $\mathrm{Ni}$ catalyst supported on activated carbon. Carbon. 1998;36(3):283-92.

6. Wang S, Zhu ZH. Effects of acidic treatment of activated carbons on dye adsorption. Dyes and Pigments. 2007;75(2):306-14.

7. Zhu ZH, Radovic LR, Lu GQ. Effects of acid treatments of carbon on N2O and NO reduction by carbon-supported copper catalysts. Carbon. 2000;38(3):451-64.

8. Malek Abbaslou RM, Tavasoli A, Dalai AK. Effect of pre-treatment on physicochemical properties and stability of carbon nanotubes supported iron Fischer-Tropsch catalysts. App. Catal. A. 2009 Feb 28;355(1-2):33-41.

9. Conte M, Davies CJ, Morgan DJ, Davies TE, Elias DJ, Carley AF, et al. Aqua regia activated $\mathrm{Au} / \mathrm{C}$ catalysts for the hydrochlorination of acetylene. J. Catal. 2012; accepted.

10. Conte M, Davies CJ, Morgan DJ, Davies TE, Carley AF, Johnston P, et al.

Modifications of the metal and support during the deactivation and regeneration of $\mathrm{Au} / \mathrm{C}$ catalysts for the hydrochlorination of acetylene. Catal. Sci. Technol. 2012 Aug 7; in press:DOI: $10.1039 / \mathrm{C} 2 \mathrm{CY} 20478 \mathrm{~A}$.

11. Hutchings GJ. Vapor phase hydrochlorination of acetylene: Correlation of catalytic activity of supported metal chloride catalysts. J. Catal. 1985 Nov;96(1):292-5.

12. Nkosi B, Coville NJ, Hutchings GJ, Adams MD, Friedl J, Wagner FE.

Hydrochlorination of acetylene using gold catalysts: A study of catalyst deactivation. J. Catal. 1991 Apr;128(2):366-77. 
13. Conte M, Carley AF, Heirene C, Willock DJ, Johnston P, Herzing AA, et al.

Hydrochlorination of acetylene using a supported gold catalyst: A study of the reaction mechanism. J. Catal. 2007 Sep;250(2):231-9.

14. Nkosi B, Coville NJ, Hutchings GJ. Vapour phase hydrochlorination of acetylene with group VIII and IB metal chloride catalysts. App. Catal. 1988;43(1):33-9.

15. Conte M, Carley A, Hutchings G. Reactivation of a Carbon-supported Gold Catalyst for the Hydrochlorination of Acetylene. Catal. Lett. 2008;124(3):165-7.

16. Zhu Y-J, Schnieders A, Alexander JD, Beebe TP. Pit-Templated Synthesis and Oxygen Adsorption Properties of Gold Nanostructures on Highly Oriented Pyrolytic Graphite. Langmuir. 2011 Sep 30;18(15):5728-33.

17. Hövel H, Becker T, Bettac A, Reihl B, Tschudy M, Williams EJ. Crystalline structure and orientation of gold clusters grown in preformed nanometer-sized pits. App. Surf. Sci. 1997 Jun 1;115(2):124-7.

18. Hövel H, Barke I. Morphology and electronic structure of gold clusters on graphite: Scanning-tunneling techniques and photoemission. Prog. Surf. Sci. 2006;81(2-3):53111.

19. Horcas I, Fernandez R, Gomez-Rodriguez J, Colchero J, Gomez-Herrero J, Baro A. WSXM: A software for scanning probe microscopy and a tool for nanotechnology. Rev. Sci. Instr. 2007 Jan;78(1):013705.

20. Carley AF, Roberts MW. An X-Ray Photoelectron Spectroscopic Study of the Interaction of Oxygen and Nitric Oxide with Aluminium. Proc. R. Soc. A. 1978 Nov 22;363(1714):403-424.

21. Carley AF, Davies PR, Jones RV, Harikumar KR, Kulkarni GU, Roberts MW. The structure of sulfur adlayers at $\mathrm{Cu}(110)$ surfaces: an STM and XPS study. Surf. Sci. 2000 Feb 20;447(1-3):39-50.

22. Beamson G, Briggs D. High Resolution XPS of Organic Polymers: The Scienta ESCA300 Database. John Wiley \& Sons; 1992.

23. Coleman VA, Knut R, Karis O, Grennberg H, Jansson U, Quinlan R, et al. Defect formation in graphene nanosheets by acid treatment: an X-ray absorption spectroscopy and density functional theory study. Journal of Physics D: Applied Physics. 2008 Mar 21;41(6):062001.

24. Harrison WA. Electronic Structure and the Properties of Solids: The Physics of the Chemical Bond. Dover Publications; 1989.

25. Stolyarova E, Stolyarov D, Bolotin K, Ryu S, Liu L, Rim KT, et al. Observation of Graphene Bubbles and Effective Mass Transport under Graphene Films. Nano Lett. 2009 Jan 14;9(1):332-7.

26. Georgiou T, Britnell L, Blake P, Gorbachev RV, Gholinia A, Geim AK, et al. Graphene bubbles with controllable curvature. Applied Physics Letters. 2011 Aug 30;99(9):093103-093103-3.

27. Shin Y-R, Jung S-M, Jeon I-Y, Baek J-B. The oxidation mechanism of highly ordered pyrolytic graphite in a nitric acid/sulfuric acid mixture. Carbon. 2013 Feb;52(0):493-8. 
28. Hathcock KW, Brumfield JC, Goss CA, Irene EA, Murray RW. Incipient Electrochemical Oxidation of Highly Oriented Pyrolytic Graphite: Correlation between Surface Blistering and Electrolyte Anion Intercalation. Anal. Chem. $1995 \mathrm{Jul}$ 1;67(13):2201-6.

29. Alsmeyer DC, McCreery RL. In situ Raman monitoring of electrochemical graphite intercalation and lattice damage in mild aqueous acids. Anal. Chem. 1992 Jul 1;64(14):1528-33.

30. Gewirth AA, Bard AJ. In situ scanning tunneling microscopy of the anodic oxidation of highly oriented pyrolytic graphite surfaces. J. Phys. Chem. 1988 Oct 1;92(20):5563-6. 
(a)

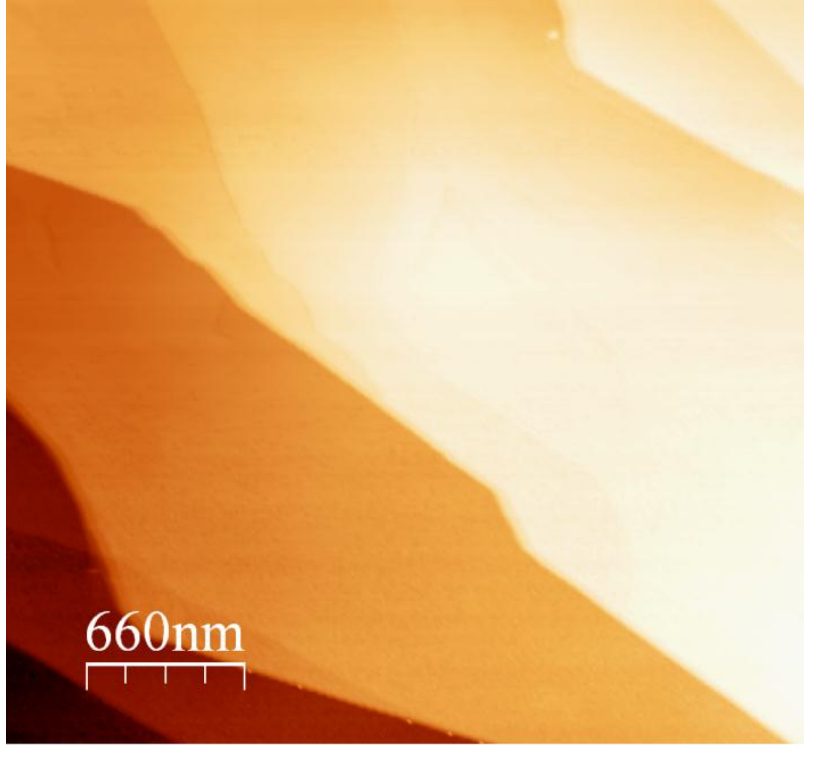

(b)
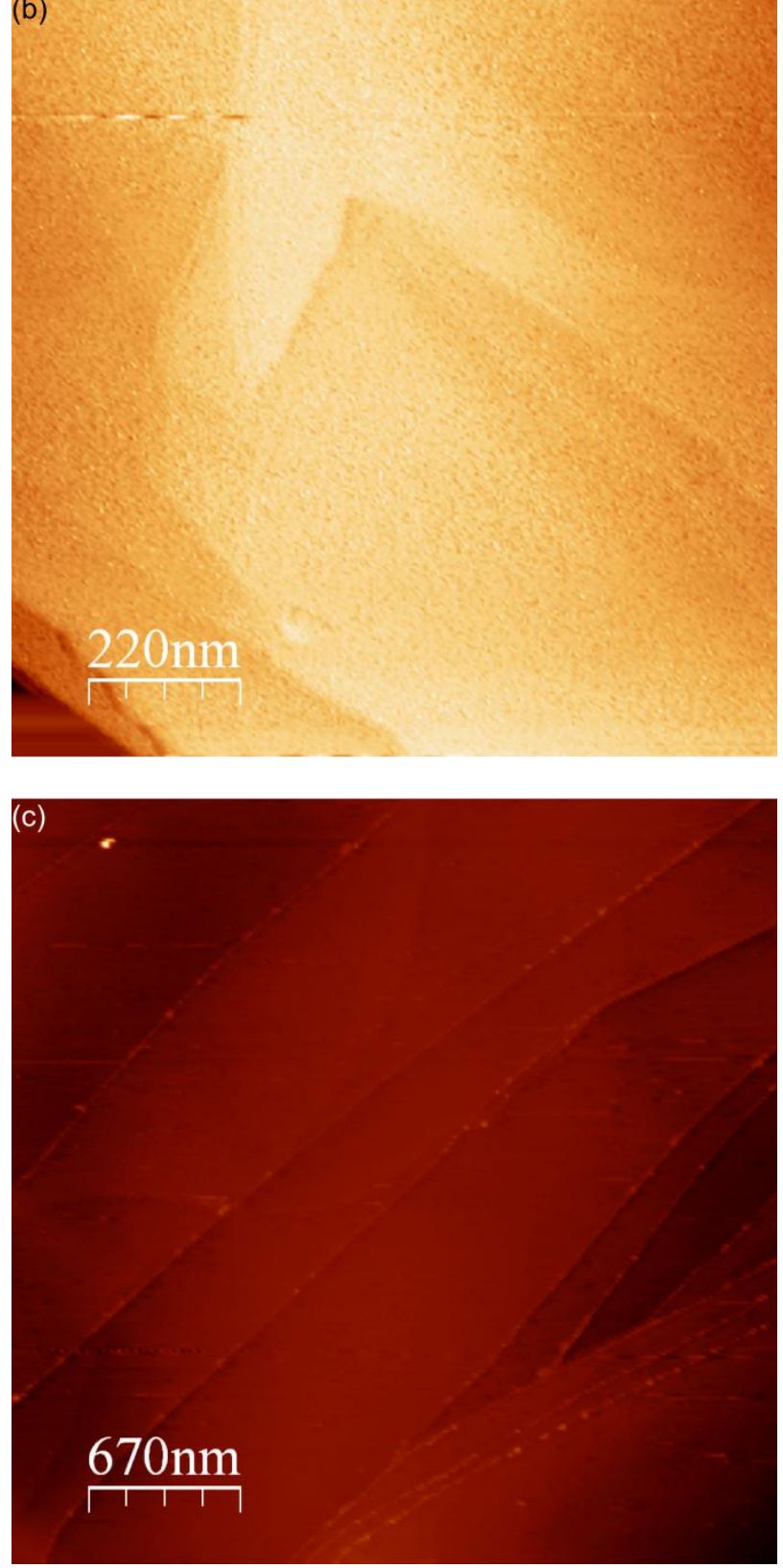

Figure 1.

(a) and (b) AFM images of clean HOPG; (c) image of HOPG treated with ultra pure water. 

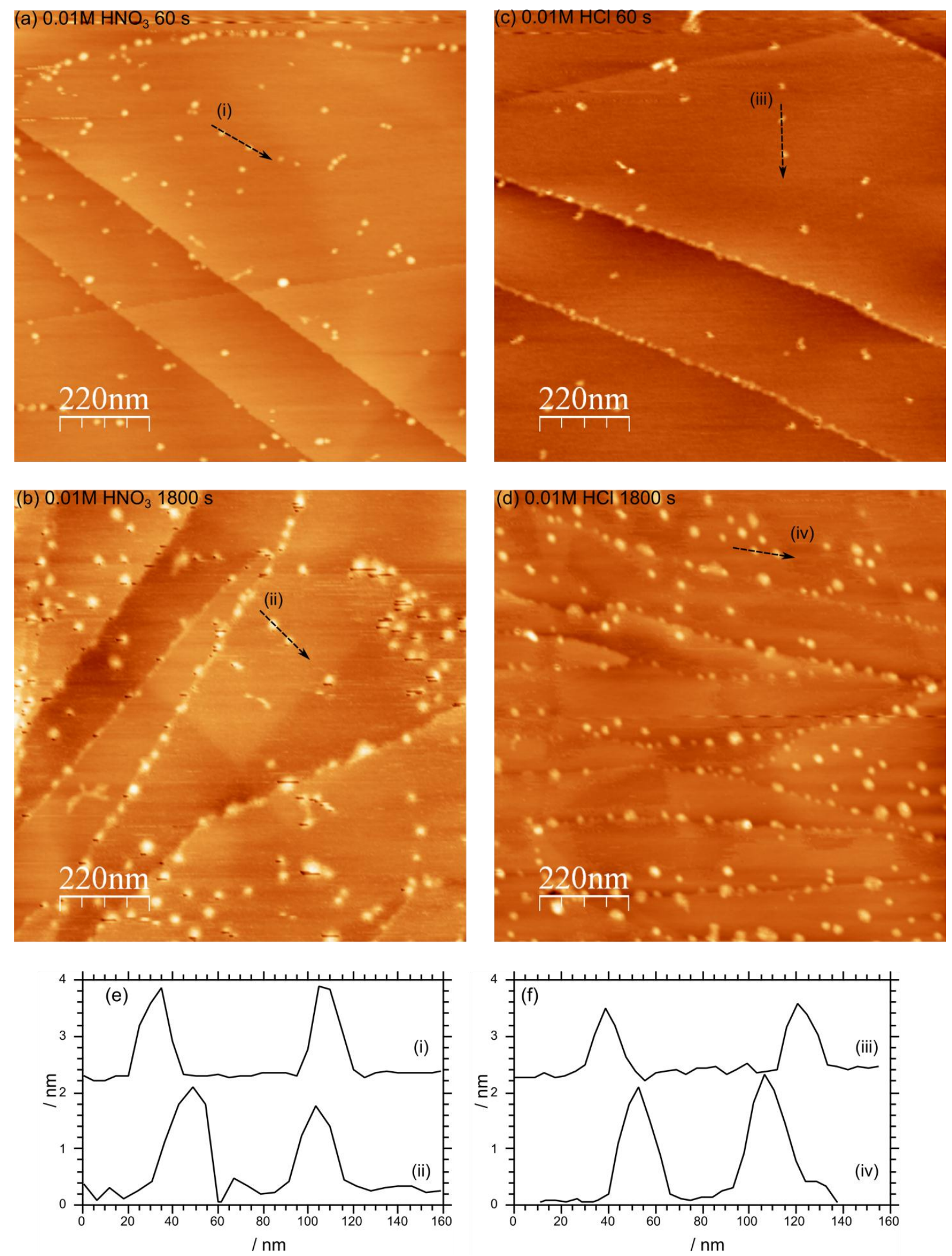

Figure 2.

AFM images of acid treated HOPG: (a) $0.01 \mathrm{M} \mathrm{HNO}_{3}$ for 60 seconds, (b) $0.01 \mathrm{M} \mathrm{HNO}_{3}$ for 1800 seconds; (c) $0.01 \mathrm{M} \mathrm{HCl}$ for 60 seconds, (d) $0.01 \mathrm{M} \mathrm{HCl}$ for 1800 seconds. Line profiles from the images are compared in (e) and (f) 

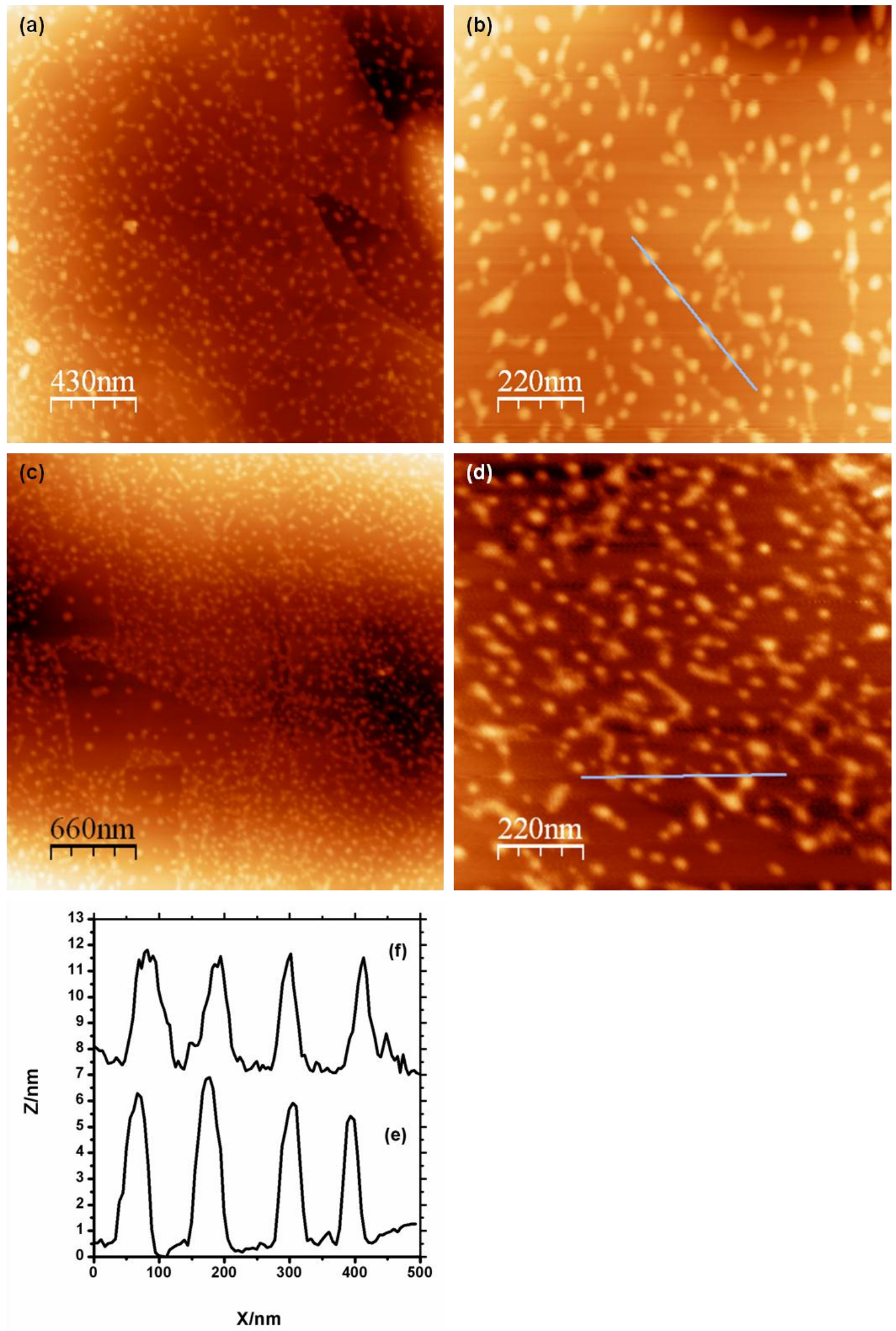

Figure 3.

AFM images of HOPG treated with $\mathrm{HCl}$ : (a) and (b) $0.1 \mathrm{M}$ for $30 \mathrm{~min}$; (c) and (d) $0.2 \mathrm{M}$ for $30 \mathrm{~min}$; (e) \& (f) line profiles from (b) and (d) respectively. 

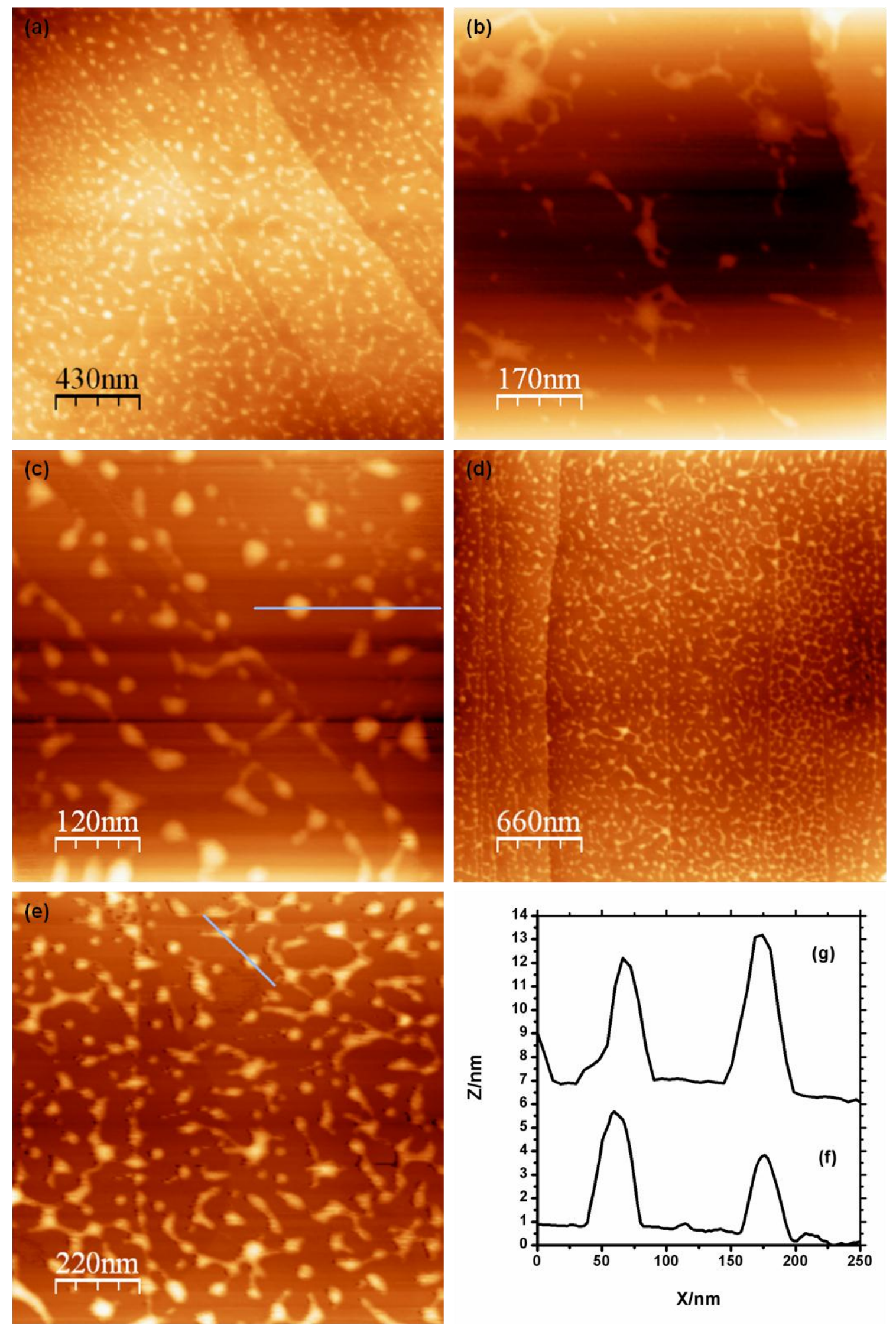

\section{Figure 4.}

AFM images of HOPG treated with $\mathrm{HNO}_{3}$; (a)-(c) $0.1 \mathrm{M} \mathrm{HNO}_{3}$ for $30 \mathrm{~min}$; (d) and (e) $0.2 \mathrm{M} \mathrm{HNO}_{3}$ for $30 \mathrm{~min}$; (f) \& (g) line profiles taken from (c) and (e) respectively. 

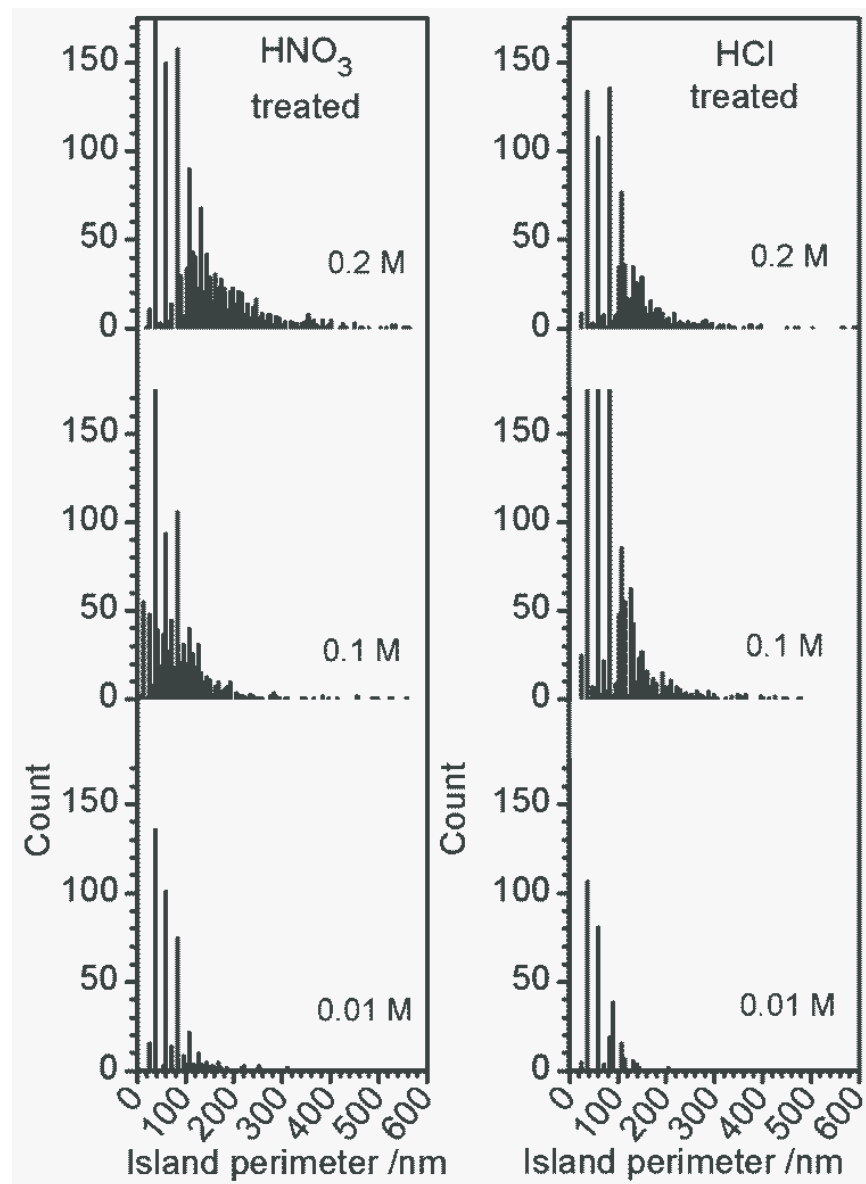

Figure 5.

The effect of acid concentration on the distribution of island sizes on graphite surfaces. Data for acid concentrations of 0.1 and 0.2 molar are offset for clarity. 

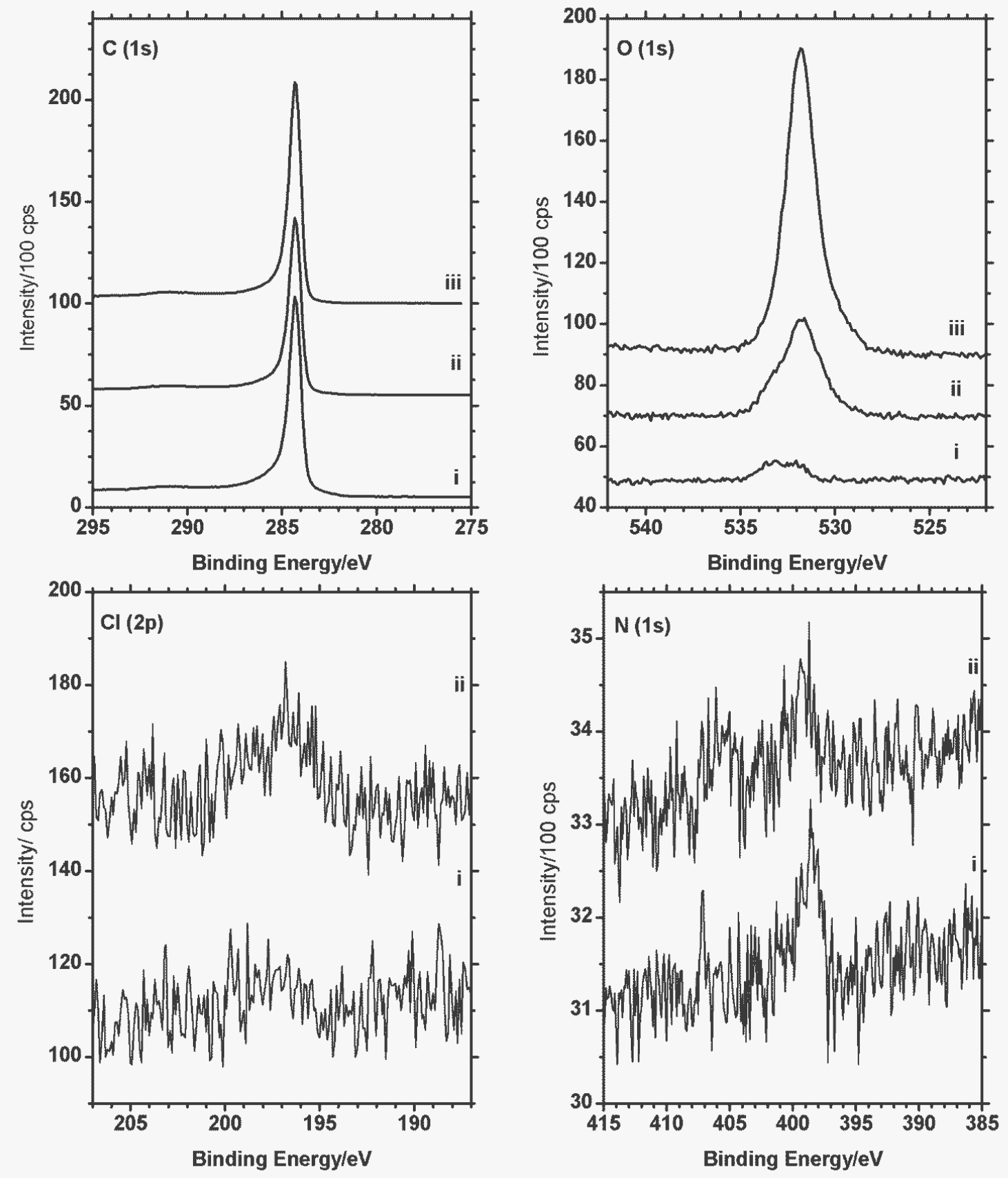

Figure 6.

XP spectra of C (1s) and O (1s) regions: (i) clean HOPG; (ii) treated with $0.2 \mathrm{M} \mathrm{HCl}$; (iii) treated with $0.2 \mathrm{M} \mathrm{HNO}_{3} . \mathrm{Cl}(2 \mathrm{p}$ ) region: (i) clean $\mathrm{HOPG}$; (ii) treated with $0.2 \mathrm{M} \mathrm{HCl}$; and N (1s) region: (i) clean $\mathrm{HOPG}$; (ii) treated with $0.2 \mathrm{M} \mathrm{HNO}_{3}$. 

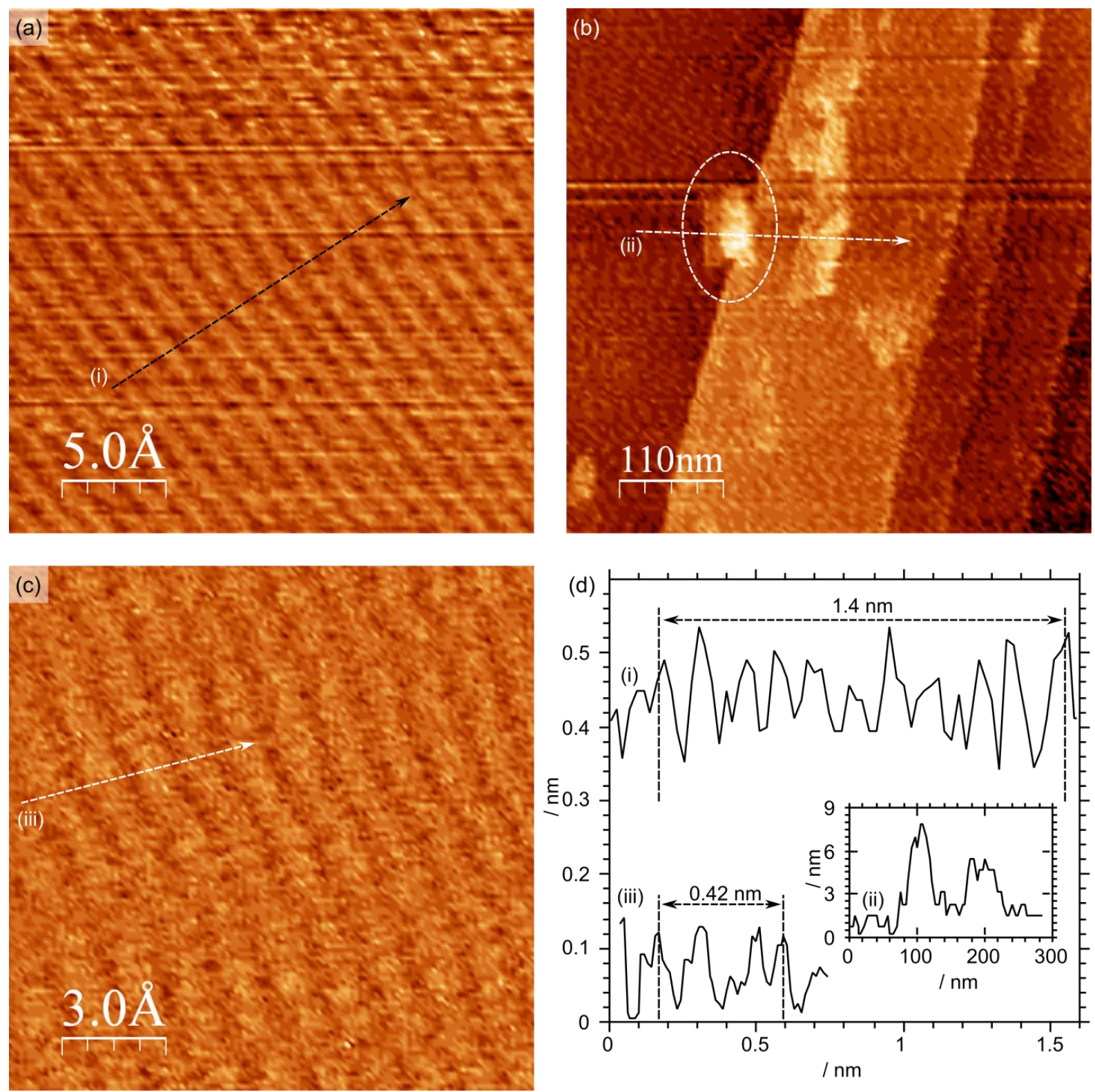

\section{Figure 7.}

STM images and line profiles of the clean and acid treated HOPG sample. (a) Clean surface showing the typical C-C periodicity of $0.14 \mathrm{~nm}$. (b) HOPG treated with $0.2 \mathrm{M} \mathrm{HNO}_{3}$, the white oval indicates a protrusion similar to those seen by AFM and confirmed by the profile shown in the inset in (d). (c) High resolution image recorded from the top of the protrusion imaged in (b), the $0.14 \mathrm{~nm}$ periodicity is again evident. 